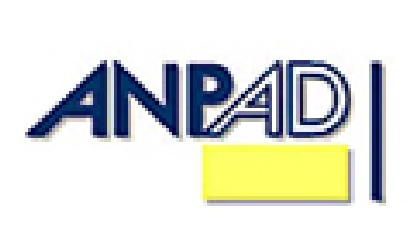

Disponível em

http://www.anpad.org.br/rac

RAC, Rio de Janeiro, v. 16, n. 3, art. 5, pp. 418-437, Maio/Jun. 2012

$(\mathrm{oc})$ EY-No

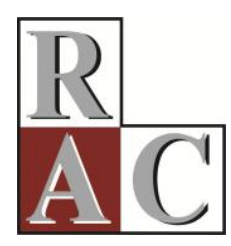

\title{
Analisando Frames Tecnológicos: um Estudo das Interpretações Sociais da Tecnologia da Informação no Contexto Organizacional
}

\section{Making Sense of Technological Frames: a Study of Social Interpretations of Information Technology in the Organizational Context}

\author{
Clarissa Carneiro Mussi * \\ E-mail: mussi.clarissa@gmail.com \\ Universidade do Sul de Santa Catarina - UNISUL \\ Florianópolis, SC, Brasil.
}

Ronaldo Zwicker (in memorian) Universidade de São Paulo - FEA/USP

São Paulo, SP, Brasil.

\footnotetext{
* Endereço: Clarissa Carneiro Mussi

Universidade do Sul de Santa Catarina - UNISUL, Programa de Pós-Graduação em Administração, Unidade Ilha-Centro, Rua Trajano, 219, Centro, Florianópolis/SC, 88010-010.
}

Copyright (C) 2012 RAC. Todos os direitos, até mesmo de tradução, são reservados. É permitido citar parte de artigos sem autorização prévia, desde que seja identificada a fonte. 


\title{
Resumo
}

Frames tecnológicos (estruturas cognitivas compartilhadas em relação à tecnologia) constituem a temática desta pesquisa. O objetivo foi analisar a natureza e a extensão de diferenças em frames tecnológicos de grupos sociais distintos, assim como compreender as razões para congruências e incongruências. A pesquisa foi realizada em uma instituição de ensino superior, e o objeto de estudo tratou-se de um sistema de informação acadêmico internacional, implementado e em uso nessa instituição. Com pressupostos epistemológicos sustentados pela perspectiva interpretativa, metodologicamente o estudo caracterizou-se por uma abordagem qualitativa. Entrevistas em profundidade, observação participante, revisão documental e artefato físico constituíram-se como as fontes de dados empíricos. Adotaram-se os fundamentos do método hermenêutico-dialético para análise e interpretação dos dados coletados. O estudo propiciou identificar que diferentes interpretações sobre o sistema de informação acadêmico são socialmente construídas pelos indivíduos inseridos em grupos sociais e fortemente afetadas pelo que acreditam, conhecem e esperam desse sistema. Características pessoais, contextuais e tecnológicas integram-se criando, reforçando e modificando frames tecnológicos, contribuindo para o delineamento de congruências e incongruências.

Palavras-chave: frames tecnológicos; tecnologia da informação; cognição social.

\begin{abstract}
Technological frames (shared cognitive structures that relate to technology) are the main theme of this research. Its general goal is to analyze the nature and extension of the differences that sustain technological frames for different social groups as well as to understand the motivations for congruencies and incongruencies. The research was conducted in a higher education institution with an international academic information system as its object of analysis. The system was already implemented at the time of the research and is in use today. Methodologically, this study is characterized by epistemological suppositions supported by an interpretative perspective and a qualitative approach. In-depth interviews, participant observation, document analysis and physical artifacts were the sources of empirical data. Data analysis and interpretation were backed up by a dialectic-hermeneutic method. The results point to different interpretations of the academic information system. These interpretations are socially constructed by the individuals and become greatly affected by their prior knowledge of the system, their assumptions and expectations towards it. Personal and contextual characteristics as well as technological ones are integrated creating, reinforcing and modifying congruencies and incongruencies in technological frames.
\end{abstract}

Key words: technological frames; information technology; social cognition. 


\section{Introdução}

A pesquisa social cognitiva pressupõe que as pessoas agem com base em suas interpretações ou representações de mundo, as quais mediam suas percepções e sua compreensão da realidade (Berger \& Luckmann, 2011; Weick, 1973). Esse pressuposto vem sendo estudado no contexto de organizações e sistemas de informação (SI), como ilustram as pesquisas de Orlikowski e Gash (1994), Davidson (2002), e Vaast e Walsham (2005). O conceito de flexibilidade interpretativa (Pinch \& Bijker, 1989), da teoria da Construção Social da Tecnologia (Social Construction of Technology [SCOT]), expressa que uma mesma tecnologia pode ter diferentes significados para diferentes pessoas, ou seja, pode estar aberta a mais do que uma interpretação. Uma falha na compreensão de pontos de vista alternativos pode conduzir a resultados não antecipados, como resistência e problemas operacionais (Symons, 1991).

Mantém relação com o conceito de flexibilidade interpretativa, o conceito de frames tecnológicos, desenvolvido e introduzido na comunidade de pesquisa em SI por Orlikowski e Gash (1994), com base na pesquisa social cognitiva e na literatura sociológica. Frames tecnológicos expressam as diferentes interpretações de uma tecnologia por diferentes grupos sociais de uma organização. Mais especificamente, frames tecnológicos são definidos como o conjunto de pressupostos, expectativas e conhecimentos sobre a tecnologia, compartilhados e mantidos coletivamente por atores de um mesmo grupo social. Com relação à tecnologia da informação (TI), há diferentes grupos sociais em uma organização - como gestores, profissionais de TI, usuários - cujos integrantes tenderão a compartilhar frames tecnológicos comuns.

Orlikowski e Gash (1994) argumentam que frames tecnológicos influenciam como os atores pensam e agem em relação à tecnologia e que, portanto, o estudo de tal assunto permite compreender as interpretações sociais da tecnologia, explicar ações e apreender significados que não seriam facilmente obtidos com outras lentes teóricas. Alguns estudos têm mostrado que diferenças significativas em frames tecnológicos de grupos sociais distintos podem refletir dificuldades em processos relacionados à tecnologia (Davidson, 2002; McGovern \& Hicks, 2004; McLoughlin, Badham, \& Couchman, 2000; Puri, 2006). A pesquisa de Orlikowski e Gash (1994), por exemplo, identificou que incongruências (diferenças) em frames tecnológicos, principalmente de usuários e profissionais da área tecnológica, conduziram a dificuldades e conflitos no desenvolvimento, implementação e uso da tecnologia.

Após a publicação do artigo de Orlikowski e Gash (1994), é limitado o número de estudos que de fato realizaram análises de frames tecnológicos empenhando-se em utilizar e desenvolver a estrutura proposta inicialmente. Embora o referido artigo tenha sido amplamente citado na literatura como uma chamada para a análise social e sócio-cognitiva de SI, poucas são as publicações referentes a alguma forma de análise de frames tecnológicos. Como argumenta Davidson (2006), citações breves do artigo ultrapassam significativamente aplicações da estrutura teórica proposta. Tem sido ressaltada na literatura a necessidade de desenvolvimento teórico que vise alcançar as contribuições potenciais que a teoria de frames tecnológicos pode trazer para o avanço do conhecimento em SI (Davidson, 2006; Puri, 2006).

A pesquisa relatada neste artigo objetivou contribuir com proposições que venham a enriquecer a teoria em questão, a partir da investigação da natureza e da extensão de diferenças em frames tecnológicos de grupos sociais distintos, assim como, da compreensão das razões para congruências (semelhanças) e incongruências (diferenças) destes em uma organização. O objeto do estudo foi um sistema de informação computadorizado específico do ambiente universitário (sistema acadêmico), ambiente este permeado por um conjunto de distintos grupos sociais (professores, alunos, gestores, profissionais de tecnologia, entre outros). A pesquisa foi orientada metodologicamente pela perspectiva interpretativa. A coleta de dados foi de natureza qualitativa, e a análise e a interpretação dos dados foram baseadas no método hermenêutico-dialético. 
A seguir são apresentados aspectos da revisão da literatura relacionada a frames tecnológicos, o referencial metodológico utilizado, a descrição e a discussão dos resultados, e as considerações finais da pesquisa.

\section{Frames Tecnológicos: Bases Teóricas}

Os estudos sócio-cognitivos e a abordagem sociológica denominada Construção Social da Tecnologia constituem-se como as raízes ou bases teóricas dos frames tecnológicos.

Cognição em organizações é uma área de pesquisa que tem despertado crescente interesse na comunidade de gestão (Walsh, 1995). A base de estudos organizacionais na perspectiva cognitiva é o indivíduo, mais especificamente sua cognição. Estes estudos consideram que os indivíduos interpretam seu ambiente e agem com base em modelos cognitivos (Tan \& Gallupe, 2003). Muitas das raízes da pesquisa cognitiva aplicada estão na psicologia, mais especificamente na psicologia cognitiva. Conceitos como estrutura de conhecimento, esquema, frame representam a preocupação de psicólogos em entender como indivíduos interpretam informações do ambiente e dão a elas significado (Walsh, 1995). Lin e Silva (2005, p. 50) definem frame como "um dispositivo cognitivo que capacita os indivíduos a compreender, entender e explicar o mundo em torno deles. Os indivíduos confiam em frames para fazer sentido de seu mundo".

A perspectiva social cognitiva estende a ideia de estruturas cognitivas individuais para grupos e organizações, sugerindo que os indivíduos de uma organização não têm somente interpretações particulares, mas também crenças compartilhadas e comuns com outros membros da organização (Orlikowski \& Gash, 1994). Surge a noção de estruturas cognitivas compartilhadas e cognição social. Neste âmbito, não se poderia deixar de referenciar os estudos das representações sociais (Moscovici, 1961), com origem na psicologia social. O conceito de frames tecnológicos tem muito em comum com esta teoria. De acordo com Vaast e Walsham (2005), ambas as noções de frames tecnológicos e representações sociais situam os indivíduos como agentes sociais. Entretanto, o conceito de representações sociais é mais amplo que o conceito de frames tecnológicos, uma vez que o primeiro refere-se às maneiras pelas quais agentes representam todos os aspectos de sua experiência e envolvimento no mundo social, enquanto o segundo está relacionado, mais especificamente, às representações da tecnologia.

A Construção Social da Tecnologia, outra abordagem cujos frames tecnológicos estão fundamentados, tem origem na publicação de Pinch e Bijker (1989) e integra uma abordagem mais ampla denominada Formatação Social da Tecnologia (Social Shaping of Technology [SST]). A SCOT caracteriza-se como uma abordagem construtivista social e, como tal, prima por um movimento contrário à simplicidade causal do determinismo tecnológico e à consideração tradicional dos impactos da tecnologia (Howcroft, Mitev, \& Wilson, 2004). Seus percursores, segundo Nijland (2004), argumentam que artefatos são socialmente construídos por grupos sociais e que sucesso ou falha não são apenas uma questão de atributos tecnológicos, mas dependem da ação interpretativa de indivíduos em seu contexto social.

\section{Frames Tecnológicos e a Pesquisa em Sistemas de Informação}

Questões sócio-cognitivas na área de SI vêm sendo abordadas desde o final da década de 70. Embora estes estudos tratem de questões sociais e cognitivas, Orlikowski e Gash (1994) apontam para a necessidade de uma articulação sistemática do papel dos frames de referência ou estruturas cognitivas no desenvolvimento e uso de sistemas. O conceito de frames tecnológicos é, portanto, introduzido pelas autoras na literatura de SI, visando investigar processos interpretativos relacionados com a TI nas organizações. 
O conceito de frames tecnológicos é utilizado para expressar as estruturas cognitivas compartilhadas ou cognições compartilhadas de uma tecnologia por diferentes grupos na organização, pressupondo que diferentes grupos sociais em uma organização podem ter diferentes esquemas interpretativos sobre uma tecnologia particular (Yoshioka, Yates, \& Orlikowski, 2002). Frames tecnológicos são definidos como o subconjunto dos frames organizacionais de atores que diz respeito aos pressupostos, expectativas e conhecimento que eles usam para compreender a tecnologia em organizações (Orlikowski \& Gash, 1994).

Além dos termos que integram a definição de frames tecnológicos, de Orlikowski e Gash (1994) - pressupostos, expectativas e conhecimento - observou-se, em outras publicações, a utilização de termos como crenças (Lin \& Silva, 2005; McLoughlin et al., 2000), percepções (Lin \& Silva, 2005; Puri, 2006), significados (Puri, 2006), background (McGovern \& Hicks, 2004), atitudes e préconcepções (Ovaska, Rossi, \& Smolander, 2005). Vaast e Walsham (2005) observam que a diferença entre as noções de atitudes ou crenças de usuários e frames tecnológicos relaciona-se ao nível de análise adotado. Focar sobre atitudes ou crenças envolve considerar o indivíduo como o ponto central. Nesta linha, enquadra-se, por exemplo, o modelo TAM, Technology Acceptance Model, amplamente conhecido e citado na literatura de sistemas de informação (Davis, Bagozzi, \& Warshaw, 1989). A noção de frames tecnológicos considera indivíduos como agentes sociais, representando seu mundo de acordo com o contexto social em que estão inseridos.

Poucos são os registros de pesquisa em sistemas de informação que, de fato, focalizaram a análise de frames tecnológicos ou desenvolveram a estrutura teórica proposta originalmente (Davidson, 2006). Estas publicações, de forma geral, envolveram a análise de frames e estavam interessadas em identificar e analisar interpretações de diferentes aspectos relacionados à tecnologia por diferentes grupos sociais. Neste sentido, são comumente analisadas diferenças e/ou semelhanças entre frames de diferentes grupos e suas implicações em processos relacionados à tecnologia, como determinação de necessidades, desenvolvimento, implementação, uso, entre outros, por exemplo, as pesquisas de Davidson, (2002); Lin e Silva (2005) e Puri (2006). A partir da identificação de diferenças em frames tecnológicos de diferentes grupos, alguns trabalhos (Lin \& Silva, 2005; Ovaska et al., 2005; Puri, 2006) analisaram como essas diferenças foram minimizadas ou resolvidas. Outros estudos (Davidson, 2002; Lin \& Silva, 2005; Ovaska et al., 2005) também buscaram compreender como e em que circunstâncias frames mudavam (deslocavam-se) no tempo e as eventuais consequências disso. Houve ainda pesquisadores (McGovern \& Hicks, 2004; McLoughlin et al., 2000) que observaram a dominância de frames tecnológicos de determinados grupos sobre outros, mostrando como processos políticos influenciavam decisões.

\section{Análise de Frames Tecnológicos: Domínio e Conteúdo}

A análise de frames tecnológicos envolve a análise da interpretação da tecnologia por um indivíduo ou grupo de indivíduos. Tal análise envolve compreender pressupostos, expectativas e conhecimentos de indivíduos sobre a tecnologia (Lin \& Silva, 2005). De acordo com Davidson (2006), a análise de frames consiste em trazer à tona pressupostos e conhecimentos profundamente mantidos por diferentes atores, sobre uma tecnologia específica, em seu contexto organizacional.

Investigar domínio e conteúdo de frames parece ser inerente à sua análise. Domínios representam elementos-chave ou categorias do frame, um conjunto de pressupostos, expectativas e conhecimentos relacionados a um tema comum, enquanto que conteúdo refere-se- ao teor de dada categoria. Como enfatizam Ovaska, Rossi e Smolander (2005) e Puri (2006), os domínios de um frame afetam como atores compreendem e interpretam questões relacionadas à tecnologia, auxiliando na identificação de alguns aspectos fundamentais da vida organizacional.

A partir da análise dos dados do estudo de campo de Orlikowski e Gash (1994) sobre a adoção de uma tecnologia de groupware, emergiram os seguintes domínios gerais de frames tecnológicos dos 
participantes daquele estudo: natureza da tecnologia (o que a tecnologia é); estratégia da tecnologia (por que a tecnologia foi introduzida); e tecnologia em uso (como a tecnologia é usada). Vários autores, como Orlikowski e Gash (1994) e Davidson (2002), concordam e alertam que domínios de frames são específicos a um contexto. Davidson (2006) observa que, em grande parte dos estudos, as denominações de domínios de frames encontrados são diferentes e seu conteúdo descrito em termos contextuais. Entretanto, argumenta que uma análise mais próxima permitiu observar que algumas similaridades têm ocorrido em estudos de casos organizacionais.

\section{Compartilhamento, Congruências e Incongruências de Frames Tecnológicos}

Os frames são socialmente construídos por meio da socialização, interação e negociação. Para Yoshioka, Yates e Orlikowski (2002), membros de um mesmo grupo compartilham papéis, experiências e conhecimento comuns em relação a uma tecnologia e, desse modo, a variância de esquemas interpretativos entre diferentes grupos tende a surgir.

O termo incongruências em frames tecnológicos é utilizado para referenciar diferenças ou discrepâncias importantes em pressupostos, expectativas e conhecimentos relacionados à tecnologia. Em sentido oposto, congruências de frames referem-se ao alinhamento, à convergência dos esquemas interpretativos de diferentes grupos sociais. O termo congruência é utilizado para indicar interpretações da tecnologia similares (domínios e conteúdo comuns), mas não idênticas (Orlikowski \& Gash, 1994).

Estudos empíricos, em geral, têm encontrado consequências negativas quando da existência de incongruências em frames tecnológicos. Barret (1999) identificou que uma das razões para os baixos índices de adoção de uma tecnologia de EDI no mercado de seguros estava associada à incongruência em pressupostos culturais mantidos por diferentes grupos. O estudo de McLoughlin, Badhan e Couchman (2000) evidenciou consideráveis problemas para criar a legitimidade em torno da adaptação e da adoção de uma nova tecnologia de produção, principalmente em decorrência de incongruência em pressupostos básicos quanto ao sistema de produção.

Yoshioka et al. (2002), em sua pesquisa empírica sobre o uso de uma ferramenta de groupware em diferentes unidades de uma organização global, encontraram diferenças nos esquemas interpretativos que ajudaram a explicar dificuldades no uso da tecnologia. Puri (2006) percebeu que a identificação de incongruência em frames divergentes de diferentes grupos ajudou a entender dificuldades relacionadas ao projeto e à implementação do sistema investigado. Neste sentido, o autor sugere que essas incongruências podem ser minimizadas e uma interpretação compartilhada da tecnologia alcançada, quando se reconhecem e se compreendem processos sócio-cognitivos, buscando-se promover interações sociais que conduzam a um consenso.

Lin e Silva (2005) atribuem incongruências em frames tecnológicos às diferentes identidades, papéis organizacionais, background e interesses de cada grupo que servem de base para a formação de sua própria interpretação da tecnologia. Para Orlikowski e Gash (1994), as incongruências podem acontecer devido a diferentes fatores, como influências políticas e falta de informação, e podem conduzir a dificuldades no uso e na mudança tecnológica. Lin e Silva (2005) observam que os frames de um grupo podem não ser aceitos por outro grupo, e que a incongruência de frames tecnológicos conduz a diferenças nas formas como estes grupos interagem com a tecnologia e a interpretam.

Segundo Davidson (2006), alinhar frames envolve trazê-los à tona e compará-los de modo que alguma compreensão comum, suficiente para ação e interação conjuntas, possa emergir. O estudo de Davidson (2002) mostra, ainda, que a incongruência pode variar com o tempo, uma vez que domínios e conteúdos de frames podem mudar em reposta a desencadeadores de mudança. Framing e Reframing são termos que vêm sendo utilizados para referenciar a mudança de frames tecnológicos. 
A noção de frames tecnológicos e seus fundamentos sugerem alguns questionamentos, investigados neste estudo, como: qual a natureza e a extensão de diferenças em frames tecnológicos de grupos sociais distintos em um ambiente organizacional acadêmico? Quais as razões para congruências e incongruências em frames tecnológicos? Como frames tecnológicos são criados, modificados ou reforçados?

\section{Metodologia da Pesquisa}

Os pressupostos epistemológicos que orientaram esta pesquisa estão fundamentados na perspectiva interpretativa. A pesquisa interpretativa pode contribuir para que pesquisadores de SI compreendam o pensamento e a ação de indivíduos em contextos sociais e organizacionais (Klein \& Myers, 1999; Walsham, 2006). Assim como outras abordagens de pesquisa, a pesquisa interpretativa não está livre de princípios e critérios a serem seguidos, que a caracterizam e nela imprimem qualidade. A condução desta pesquisa pautou-se nos princípios e critérios definidos por Klein e Myers (1999) para condução e avaliação de estudos de campo interpretativos em sistemas de informação. A perspectiva interpretativa foi associada à abordagem qualitativa (Strauss \& Corbin, 2008). O tipo de pesquisa caracterizou-se como estudo de caso único incorporado (Yin, 2009). Único por se tratar de uma única organização (uma universidade), e incorporado porque envolveu subunidades de análise (grupos sociais).

\section{Contexto e sujeitos da pesquisa}

A pesquisa foi realizada em uma universidade de grande porte, sem fins lucrativos e com 50 anos de existência. Esta universidade apresenta uma história bastante singular no que se refere à adoção e à implementação de sistemas de informação. Com relação a sistemas de informação acadêmicos, fazem parte da história da universidade três sistemas: o primeiro, desenvolvido internamente e utilizado durante 12 (doze) anos; o segundo, um sistema de grande porte, de um fornecedor de softwares acadêmicos nacional, utilizado durante 2 (dois) anos; e o terceiro, foco desta pesquisa, um sistema de grande porte, de um fornecedor de softwares acadêmicos internacional (americano). O projeto de implementação deste último sistema teve duração de 9 (nove) meses, abrangendo as áreas acadêmica e financeira da universidade. Compunham o projeto em torno de 30 (trinta) pessoas, com dedicação full time. Foram implementados os seguintes módulos: Student Records/GradeBook, Student Financials, Financial AID, Academic Advisement Campus Community/Campus Self Service, Recruiting and Admissions.

A identificação dos grupos sociais integrantes desta pesquisa observou os critérios propostos por Orlikowski e Gash (1994), sendo definidos de forma a agregar em cada grupo social indivíduos que compartilham papéis, background, experiências e conhecimentos comuns, e que estabelecem um considerável nível de interação entre si. Esses critérios, em conjunto com o conhecimento prévio do ambiente de pesquisa pelos pesquisadores, permitiram identificar seis grupos sociais que foram investigados: coordenadores de curso, professores, alunos, pessoal técnico-administrativo, diretores e profissionais de sistemas de informação. Para fins de inclusão dos sujeitos da pesquisa em cada um dos grupos, foram definidos critérios específicos. Ao total, participaram da pesquisa 48 sujeitos, distribuídos igualmente entre os grupos sociais, isto é, oito pessoas em cada grupo.

\section{Coleta de dados}

A perspectiva interpretativa, a abordagem qualitativa e o estudo de caso baseiam-se em uma ampla variedade de fontes de evidência. Nesta pesquisa, evidências empíricas foram coletadas a partir de um conjunto de fontes e técnicas: entrevistas em profundidade, observação participante, análise documental e análise de artefato físico. 
As entrevistas realizadas tiveram como suporte um roteiro elaborado sob a perspectiva dos objetivos e fundamentos teóricos da pesquisa. No início da entrevista, foram apresentados ao participante o objetivo da pesquisa e os princípios éticos a ela pertinentes. Esses princípios constavam de um termo de consentimento livre e esclarecido, em que o entrevistado declara estar esclarecido sobre os objetivos e procedimentos da pesquisa, concordando em participar desde que respeitados os princípios éticos a ele apresentados. As entrevistas foram realizadas durante quatro meses, cada uma teve duração de 40 a 120 minutos, e produziram cerca de 29 horas de gravação e 620 páginas de transcrição.

Como um dos pesquisadores exercia atividades na instituição, para a coleta de dados foi também utilizada a técnica da observação participante (Nandhakumar \& Jones, 2002). A prévia interação social desse pesquisador com o contexto pesquisado facilitou a anotação de passagens relevantes (conversas informais e comportamentos) relacionadas ao foco de estudo, o que enriqueceu os dados coletados. Da mesma forma, essa interação também contribuiu para uma melhor interpretação dos dados nas análises realizadas.

Ainda neste estudo, documentos referentes aos processos de avaliação dos sistemas, relatórios gerados por sistema, site da universidade, jornal interno e comunicações eletrônicas, são alguns exemplos de dados secundários que foram analisados. Outra técnica de coleta de dados, citada por Yin (2009), que pode ser utilizada em um estudo de caso, consiste na análise de artefatos físicos, como uma tecnologia, uma ferramenta ou um instrumento, uma obra de arte. A tecnologia da informação, foco desta pesquisa, consiste em um artefato físico utilizado como fonte de dados. Vale assinalar que a interação dos pesquisadores com o sistema acadêmico em uso na instituição permitiu não somente considerá-lo como fonte de dados, mas também compreender melhor a perspectiva dos entrevistados quando se referiam ao sistema.

\section{Análise de dados}

Como os pressupostos epistemológicos que sustentam esta pesquisa têm por base a perspectiva interpretativa, a análise e a interpretação dos dados coletados pautaram-se em fundamentos do método hermenêutico-dialético (Cole \& Avison, 2007; Minayo, 2008). Seguiram-se as diretrizes gerais propostas por Minayo (2008) para a operacionalização de tal método. Essas diretrizes estão organizadas nas seguintes etapas: (a) ordenação dos dados constituída pela transcrição das gravações, dos dados da observação participante por fonte e dos documentos por assunto; (b) classificação dos dados envolvendo a constituição dos corpus de comunicação, análise transversal e categorização, identificação das grandes categorias e estabelecimento de diferentes níveis de classificação e análise; (c) análise final incluindo a interpretação entre grupos sociais e a articulação do material empírico analisado com o referencial teórico.

No caso deste estudo, no que se refere à classificação dos dados, constituíram-se seis corpus representando os seis grupos sociais. A leitura transversal envolveu a análise, em diferentes níveis, de todas as entrevistas no âmbito de cada grupo, abrangendo um estudo aprofundado, detalhado e exaustivo de cada corpus, com base no procedimento de categorização. As categorias preliminares ou pré-estabelecidas, aqui rotuladas de grandes categorias de análise, originaram-se das teorias de base desta pesquisa. Inicialmente, procedeu-se à leitura das entrevistas objetivando identificar o que se caracterizava como frames, com base no conceito de Orlikowski e Gash (1994). O primeiro nível de análise envolveu a busca das grandes categorias: conhecimentos, pressupostos e expectativas. A partir das definições destes termos, encontradas em dicionários das áreas de psicologia, sociologia e filosofia, e considerando publicações com foco na análise de frames tecnológicos, tais como Davidson (2002), Lin e Silva (2005), e Puri (2006), considerou-se como:

conhecimentos - o que o sujeito ou grupo social conhece (as informações conservadas ou assimiladas por um sujeito);

pressupostos - o que o sujeito ou grupo social conjetura, presume, supõe; em que acredita; 
expectativas - o que o sujeito ou grupo social espera; esperanças.

Posteriormente, no segundo nível de análise, para cada uma das grandes categorias, a partir de leituras e releituras de cada entrevista, identificaram-se categorias emergentes, representando, de formal geral, os domínios dos frames tecnológicos encontrados (tecnologia na prática, gerenciamento do sistema, desenvolvimento e implementação). No terceiro nível de análise, foram identificadas subcategorias para cada uma destas categorias/domínios (conhecimentos, pressupostos e expectativas sobre tecnologia na prática; conhecimentos, pressupostos e expectativas sobre gerenciamento do sistema; conhecimentos, pressupostos e expectativas sobre desenvolvimento e implementação). A partir destas subcategorias, emergiram novas categorias e assim sucessivamente, sendo o número de níveis de análise variável, de acordo com cada corpus.

\section{Descrição e Discussão dos Resultados}

Esta seção aborda a natureza dos frames tecnológicos dos grupos sociais da organização em estudo, apresentando os domínios de frames encontrados; aborda as congruências e incongruências de frames identificadas e analisa as suas razões.

\section{Natureza dos frames tecnológicos}

O caráter coletivo de estruturas cognitivas disseminadas entre grupos sociais para observar, interpretar, atribuir significado e utilizar a tecnologia (Lin \& Silva, 2005) é o requisito primordial para se rastrear um frame tecnológico. De fato, as interações sociais entre os indivíduos moldam sua visão e interpretação da realidade vivida no espaço socio-organizacional que eles ocupam. $\mathrm{O}$ discurso dos atores entrevistados no campo de pesquisa geralmente não é individual, mas traz a voz do coletivo, revelando-se produto dessas interações sociais, como ilustram os trechos: "Eu vejo que outros coordenadores pensam da mesma forma" (coordenador de curso); "todo mundo criou uma super expectativa, tu entendes?" (professor); "a maior dificuldade nossa é que, em momento nenhum nós fomos ouvidos" (professor); "quando a gente tenta levar estas angústias que tem hoje, a gente não consegue ter a receptividade que gostaria, infelizmente" (técnico-administrativo).

Um conjunto de pressupostos, expectativas e conhecimentos correspondentes a um tema comum - a partir deste conceito de domínio de um frame tecnológico se sucedeu à investigação analítica da natureza das estruturas interpretativas compartilhadas na instituição. Os principais domínios de frames detectados na análise dos dados foram três: tecnologia na prática; gerenciamento do sistema; desenvolvimento e implementação. Esses domínios emergiram dos depoimentos dos participantes da pesquisa, sem que houvesse pré-definições das categorias na análise de dados, em respeito à natureza ideográfica dos frames, que, como enfatiza Davidson (2006), são definidos dentro de um contexto específico de pesquisa.

As interpretações dos grupos sociais da pesquisa a respeito da utilização diária do sistema que eles e outros grupos fazem ou farão correspondem ao núcleo central do domínio tecnologia na prática. As consequências e condições prováveis ou reais associadas ao uso da tecnologia prioridades e recursos, facilidade de uso, imagens das pessoas quanto ao sistema e a compreensão de suas capacidades e funcionalidades - são temas da atenção dos entrevistados. Nos discursos dos atores organizacionais encontraram-se também várias declarações sobre a forma como o sistema acadêmico é gerenciado pela instituição. O domínio gerenciamento do sistema trata, em síntese, da compreensão que as pessoas têm das ações e desdobramentos potenciais ou factuais associados ao esforço de gestão da tecnologia. $\mathrm{O}$ domínio referente ao desenvolvimento e implementação do sistema acadêmico, por sua vez, abarca entendimentos apresentados pelos diversos grupos sociais a respeito da fase que precedeu o início da efetiva utilização da tecnologia por seus usuários, e também dos desenvolvimentos que têm sido realizados. Trata-se, então, de conhecimentos, pressupostos e expectativas mantidos pelos grupos sobre a estratégia de desenvolvimento, bem como sobre as 
motivações que explicam a decisão de troca de sistema, os critérios de sua seleção, seu custo, o projeto de desenvolvimento (parametrização e customização), a implementação da tecnologia e a participação de grupos sociais nesses processos.

Entre os temas emersos dos discursos analisados, há diferentes graus de concordância e discordância entre indivíduos e grupos. Assim, procede-se à análise de congruências e incongruências nos frames tecnológicos (conhecimentos, pressupostos e expectativas) dos grupos sociais pesquisados, a fim de se apreciar a extensão das diferenças e semelhanças de elementos cognitivos centrais em cada um dos três domínios genéricos acima descritos.

\section{Congruências e incongruências em frames}

Consideradas as categorias de conteúdo do frame tecnologia na prática $\left(1^{\circ}\right.$ domínio de frame identificado), observam-se interpretações bastante semelhantes compartilhadas pelos atores organizacionais e seus grupos. Percebe-se que os grupos sociais da instituição guardam imagens muito similares do período inicial de uso do sistema acadêmico. Suas representações são negativas e descrevem problemas de funcionamento e utilização da tecnologia naquela fase. A falta de conhecimento para a plena utilização do sistema acadêmico em seu estágio inicial de operação é percebida consensualmente pelos diversos grupos. Alguns, como os de coordenadores, professores e direção, concordam que ainda há deficiência de saber como utilizar integralmente todas as funcionalidades oferecidas pelo sistema, atribuindo à falta de capacitação de usuários o motivo gerador dessa situação.

Outro aspecto comum aos grupos quanto à utilização do sistema na prática é a forma como aprenderam e aprendem a operá-lo: iniciativas autônomas de aquisição de habilidades e conhecimentos sobre a tecnologia, através do próprio uso, da ajuda de pares e de busca de informações suprem as deficiências do treinamento oferecido aos usuários. $\mathrm{O}$ grupo de profissionais de sistemas, por sua posição hierárquica, atribuições de gerenciamento e manutenção do sistema, apresenta um perfil diferenciado dos demais grupos usuários do sistema. Questões relativas à interação sistemausuário na sua prática cotidiana de trabalho não estão no foco de seus discursos. Os conhecimentos dos grupos de professores, alunos e colaboradores técnico-administrativos quanto ao uso da tecnologia revelam congruência quanto ao entendimento de que o sistema vem sendo aprimorado ao longo do tempo, resultando na redução de problemas nas funcionalidades.

Sobre os pressupostos concernentes ao domínio tecnologia na prática, os grupos sociais apresentam várias congruências e incongruências em seus frames. Quando tratam dos motivos que afetam a frequência do uso do sistema, principalmente por coordenadores, professores e alunos, todos os grupos citam como um dos fatores a resistência à mudança, característica peculiar e natural do comportamento humano. Para a maior parte dos dirigentes e profissionais da área de sistemas, esse é o principal detonador das críticas negativas e da não adoção efetiva do sistema nas práticas de trabalho dos usuários. Incongruentemente, colaboradores técnico-administrativos, alunos, professores e coordenadores arrolam outros tantos fatores que concorrem para essa situação como, por exemplo, falta de usabilidade, pouca confiabilidade no sistema, limitações institucionais quanto à infraestrutura, baixa velocidade de processamento, falta de informações sobre a tecnologia.

Quanto à identificação das causas de problemas iniciais na operação do sistema, os profissionais de sistemas elencam fatores como erros de operação, erros de dados dos sistemas anteriores (nesse aspecto também concordam os dirigentes), falhas de configuração do sistema e escassez de tempo para a condução da fase de projeto. Os grupos de alunos, professores, colaboradores técnicoadministrativos e dirigentes citam vários outros motivos diversos entre si, mas pressupõem consensualmente que a falta de capacitação foi um dos geradores de erros e dificuldades no início da operação do sistema.

Um frame congruente nos grupos de professores, coordenadores e colaboradores técnicoadministrativos aborda os efeitos danosos da centralização, entendida aqui como as restrições específicas de acesso às funcionalidades do sistema impostas aos diversos grupos sociais, sob dois 
aspectos. Para os docentes, essa centralização gera falta de conhecimento mais amplo do software acadêmico, o que acaba afetando a interação usuário-sistema. Na perspectiva dos coordenadores e colaboradores técnico-administrativos, devido às restrições de acesso enfrentadas pelos setores técnico-administrativos, sua eficiência no atendimento às demandas estudantis fica comprometida, já que a dependência desses setores a ações de outras esferas institucionais provoca morosidade nos retornos (feedbacks) aos alunos.

Os grupos de direção, profissionais de sistemas e colaboradores técnico-administrativos concordam que os problemas atualmente vividos em relação ao uso do sistema na prática são mais ligados à ação humana do que à própria tecnologia. Para os profissionais de sistemas, por exemplo, outros aspectos, como traços da cultura organizacional e do estilo de gestão, erros de operação e de dados legados dos sistemas anteriores, assim como a realização de alguns processos internos, diferentemente da forma prevista no sistema, explicam as dificuldades presentes. Os colaboradores técnico-administrativos concordam que erros de operação causam problemas, mas reforçam que, além disso, o desconhecimento da tecnologia vigente, a falta de comprometimento de alguns usuários, a falta de conscientização quanto à integração da atuação dos setores promovida pelo sistema e fluxos mal ou não definidos também trazem situações indesejadas e problemas. Integrantes do grupo diretivo citam falta de conhecimento do sistema pelos usuários, principalmente dos processos como um todo. Citam também dificuldades de docentes com informática, dificuldade de mudança de hábito em relação à forma de trabalho anterior e falta de sensibilização e conscientização dos usuários para a importância do sistema no contexto organizacional como sendo os fatores que explicam os problemas atualmente vividos em relação ao uso do sistema na prática.

Quanto às expectativas dos grupos sociais, genericamente, pode-se argumentar que coordenadores, professores, alunos e colaboradores técnico-administrativos almejam melhorias no sistema. É interessante notar que ocorre um descompasso do frame de tecnologia na prática referente aos pressupostos de alguns diretores e profissionais de sistemas e os pressupostos e expectativas da maioria dos coordenadores, professores e alunos quanto às suas convicções em relação à tecnologia e às suas melhorias. Se os primeiros acreditam nas potencialidades do sistema, que o mesmo tem melhorado continuamente, e que por isso reduziram-se as críticas, os segundos apresentam dúvidas em relação às potencialidades da tecnologia e afirmam ainda aguardar por várias melhorias.

De modo geral, os participantes reconhecem a relevância de ações de gerenciamento do sistema ( $2^{\circ}$ domínio de frame identificado) e de setores integrantes dessa área para o estado da arte do uso, tanto quanto a aspectos técnicos (programações, manutenção preventiva e corretiva, políticas de segurança e qualidade dos dados) como quanto aos ligados aos profissionais atuantes no setor (capacitação técnica, competência, conhecimento sobre o contexto organizacional e os serviços de apoio e treinamento que prestam aos usuários do sistema).

Existe congruência entre coordenadores, professores e alunos sobre a falta de conhecimento acerca do setor formalmente responsável pelo gerenciamento do sistema, de sua estruturação e da organização dos serviços que presta aos usuários. Esses mesmos grupos concordam ainda que a comunicação entre a área de gerenciamento do sistema e demais setores e agentes organizacionais é precária e ineficiente. Por outro lado, os grupos de colaboradores técnico-administrativos, direção e profissionais de sistema convergem entre si, e divergem dos demais, quanto à identificação do setor responsável pela gestão tecnológica na instituição. Tal identificação não é, contudo, tão precisa para os grupos de colaboradores técnico-administrativos e direção no que tange ao reconhecimento de atribuições e serviços prestados pela estrutura de apoio ao sistema. O grupo de profissionais de sistemas, como diretamente envolvido no gerenciamento da área de tecnologia, tem completa clareza sobre suas funções hierárquicas, atribuições e serviços prestados.

Quanto às pressuposições dos grupos sociais acerca do domínio gerenciamento do sistema, observa-se que a posição do setor de tecnologia da informação na estrutura organizacional, suas atribuições e sua atuação fazem com que o grupo de profissionais de sistemas mantenha pressupostos marcantemente distintos dos demais. Seu papel-chave na condução do gerenciamento do sistema na organização o coloca, de antemão, do lado de dentro do balcão no trato de questões relativas à gestão 
do software. Por exemplo: sobre o tema da capacitação de usuários, profissionais da área de sistemas acreditam que a instituição e sua alta administração precisam comandar um amplo processo que envolva, além do próprio setor de tecnologia, outros setores da Universidade, uma vez que aqueles não devem ser os únicos responsáveis pelas iniciativas de capacitação. Por outro lado, grupos como o de colaboradores técnico-administrativos, professores, coordenadores e alunos apontam os profissionais de sistemas como responsáveis pelas falhas e deficiências dos programas de capacitação empreendidos. Ainda quanto à atuação da área de sistemas, seus profissionais afirmam adotar uma postura proativa, e não totalmente reativa na resolução de problemas; os colaboradores técnicoadministrativos, entretanto, dizem ser necessária uma atuação mais preventiva na gestão do sistema.

No domínio de gerenciamento do sistema, detectaram-se várias congruências entre coordenadores de curso, docentes e discentes. Eles concordam que, para além de falhas intrínsecas da ferramenta tecnológica, os gestores de sistemas são, sim, responsáveis pelos problemas ocorridos na operação do sistema. Alegam que a sua falta de participação (dos grupos) no processo de aprimoramento do sistema resulta no desconhecimento de suas reais necessidades, que nem sempre são atendidas, e que isso causa problemas no uso que fazem dele. Coordenadores, professores e colaboradores técnico-administrativos também convergem no entendimento de que as restrições de acesso ao sistema que enfrentam, e que estariam sob o jugo do setor de gerenciamento, afetam negativamente suas práticas de trabalho.

Os grupos sociais consentem integralmente quanto à identificação da necessidade de se realizarem programas de capacitação que atinjam a todos os públicos que interagem com o sistema: usuários finais, gestores, profissionais da área de sistemas. Docentes, coordenadores e colaboradores técnico-administrativos esperam que o setor de gerenciamento da tecnologia estabeleça melhor comunicação com os diversos setores da instituição. Alinham-se a esse entendimento as expectativas de colaboradores técnico-administrativos e dirigentes (de nível médio / uso direto) de que haja mais feedback a usuários sobre causas de problemas que enfrentam no sistema e encaminham para resolução pelo setor de tecnologia da informação.

Ao declararem suas impressões sobre o período de desenvolvimento e implementação $\left(3^{\circ}\right.$ domínio de frame identificado) do sistema, os grupos sociais retrocedem no tempo para reconstituir a época de mudança tecnológica. Eles mostram-se desalinhados em seus conhecimentos sobre esse tema. Coordenadores, professores, alunos e a maioria dos colaboradores técnico-administrativos não têm clareza sobre as razões para a troca de sistemas e nem de como se procedeu à seleção e à decisão de aquisição do novo sistema. Em contraponto, a maioria dos membros do grupo direção e dos profissionais de sistemas conhece como transcorreu esse processo. O grupo desses profissionais apresenta elevada proficiência de conhecimentos sobre todas as fases do ciclo de vida do sistema e a maioria dos integrantes do grupo diretivo, apesar de não conhecer tão bem o referido processo, alinhase às posturas desses profissionais. Entretanto, é marcante a incongruência de frames entre o grupo de profissionais de sistemas e os de coordenadores, professores e alunos quanto às categorias de conteúdo desse domínio.

Outra convergência de frames de coordenadores, professores e alunos refere-se à não participação desses grupos nas etapas de seleção, desenvolvimento e implementação do sistema. Quando expressam seus pressupostos, coordenadores, professores e alunos acreditam basicamente nas mesmas razões para que houvesse ocorrido a troca de sistema: limitações do software anterior quanto à integração acadêmico-financeira, limitações ao suporte do crescimento da Universidade e melhor atendimento aos usuários. Já os colaboradores técnico-administrativos apresentam uma compreensão mais pragmática sobre a mudança tecnológica, atribuindo-a aos muitos problemas ocorridos durante a operação do sistema anterior. Sobre sua influência na decisão de mudança e na seleção do novo sistema, coordenadores, professores, alunos e a maioria dos colaboradores técnico-administrativos entrevistados sustentam que não tiveram qualquer influência direta na troca e na seleção do sistema. Apenas alguns colaboradores acreditam poder ter exercido influência indireta naquele processo, em função das reclamações que apresentavam sobre o sistema anterior. 
A estratégia de desenvolvimento do sistema é um tema também relevante no discurso dos grupos sociais pesquisados. Se a maioria dos coordenadores e professores concorda em uníssono que a Universidade deveria ter optado por desenvolver o sistema internamente, com seu próprio expertise, os profissionais de sistemas divergem deles afirmando que não haveria condições para isso por falta de estrutura tanto de pessoal quanto tecnológica para o intento, além de outros motivos. Divergências internas são observadas entre os membros do grupo direção quanto a essa questão. Alguns gestores de nível médio tendem a crer que a estratégia de se desenvolver internamente o sistema conduziria à observância de características peculiares da instituição negligenciadas por uma tecnologia externa. Esse argumento é contraposto por alguns dirigentes de alto nível, que acreditam serem as instituições de ensino superior muito semelhantes, o que possibilita a aquisição de um software externo, estratégia ainda mais recomendável quando não se dispõe de infraestrutura interna para o desenvolvimento. E a estratégia de desenvolvimento adotada é ainda questionada, indiretamente, pelos colaboradores técnico-administrativos, quando afirmam que uma das causas para os problemas de uso da tecnologia é sua origem estrangeira, que dificulta sua adaptação à realidade institucional.

As expectativas relatadas pela maior parte dos grupos sociais pesquisados sobre o período de troca, desenvolvimento e implementação do sistema acadêmico convergem para o otimismo quanto às potencialidades de melhorias organizacionais a serem trazidas pela tecnologia. Embora se encontre uma divergência de interpretação mantida por parte de alguns integrantes do grupo dos coordenadores, que duvidava das promessas de sucesso do novo sistema, os demais grupos declaram ter esperado pelo aprimoramento de processos com a adoção deste.

\section{Compreendendo o porquê das congruências e incongruências em frames tecnológicos}

$\mathrm{Na}$ análise de congruências e incongruências de frames entre grupos sociais e dentro de cada grupo, devem-se considerar as especificidades do contexto organizacional e das características dos atores que compõem seu elenco. Detectar diferenças de saber, contextuais e do aparato tecnológico pode facilitar a compreensão de divergências em frames. Considerando-se que cada grupo social tem acesso a determinadas e específicas funcionalidades da ferramenta tecnológica, que as formações e experiências profissionais dos membros dos grupos sociais são variadas, que suas atuações organizacionais são orientadas pela jurisdição de seus setores e cargos, pergunta-se: o sistema acadêmico, como objeto de interpretação, é estável e oferece iguais possibilidades de observação a todos os atores e grupos sociais da organização? Provavelmente não, e isso parece explicar e ressaltar, em parte, as congruências e incongruências de frames identificadas. Se as diferenças são mais claramente justificadas pela heterogeneidade de conhecimentos, experiências e atribuições, as congruências de frames apontam para os limites de flexibilidade interpretativa impostos pelas propriedades técnicas do sistema de informação (Doherty, Coombs, \& Loan-Clarke, 2006) e para a interferência do contexto na viabilização de processos de socialização, interação, ou negociação (Orlikowski \& Gash, 1994). Ou seja, se mesmo com tantos fatores intervenientes nos esquemas interpretativos, observa-se forte consenso nos diferentes grupos sociais a respeito de certos conhecimentos, pressupostos e expectativas sobre o artefato tecnológico, conclui-se, em parte, que dele emanam limitadores desses mapas cognitivos coletivos. Por exemplo, todos os grupos sociais apresentam expectativas quanto a melhorias na usabilidade do sistema acadêmico, o que faz crer que ele, de fato, seja dotado de características que enrijecem a flexibilidade de interpretações sobre este atributo.

Os papéis desempenhados pelos grupos sociais integrantes da organização também afetam os esquemas compartilhados de intersubjetividade, realçando a influência do contexto nas possibilidades que os grupos têm de socialização, interação e negociação. A estrutura hierárquica e suas implicações (unidade e cadeia de comando, poder decisório, atribuições setoriais, canais formais de comunicação e acesso a informações), as interações sociais formais e informais, a cultura organizacional e os recursos físicos e tecnológicos disponíveis concorrem para viabilizar, restringir e moldar oportunidades de aquisição e compartilhamento de conhecimentos, formulação de pressupostos e de expectativas de um grupo social. Se representações sociais são elaboradas (framing) e reelaboradas (reframing) quando os atores representam seu ambiente, e se isso depende de seu engajamento nesse ambiente, e das 
respostas que percebem de tal engajamento, como afirma Valsiner (2003), as possibilidades de atuação dos grupos sociais numa organização, oferecidas ou norteadas por sua estrutura e dinâmica, são particularmente interferentes na sustentação da lógica dominante de um dado grupo de indivíduos. Esse entendimento pode ajudar a explicar incongruências de frames tecnológicos de grupos distintos, como também a observação de posicionamentos antagônicos dentro de um mesmo grupo. A partir de esquemas cognitivos estabelecidos, da capacidade reflexiva de cada grupo e de seus membros, operantes sobre as experiências vividas num contexto organizacional ao longo de um eixo temporal, os atores renovam suas interpretações e transformam as representações compartilhadas e suas ações (Moscovici, 1981; Vaast \& Walsham, 2005).

O poder prescritivo do contexto organizacional sobre os frames parece residir em sua função estruturante na composição desses grupos sociais: membros de um mesmo grupo compartilham papéis, experiências e conhecimento quanto a uma tecnologia e, assim, a variância de esquemas interpretativos entre diferentes grupos tende a surgir (Pinch \& Bijker, 1989; Yoshioka et al., 2002). Ao se aventar a hipótese de frames de grupos distintos convergirem, há que se investigar os contornos das interações que ocorrem entre eles. Se há grandes dificuldades de comunicação, e, portanto, de socialização de conhecimentos, pressupostos e expectativas entre professores e alunos de um lado, e profissionais de sistemas e alta direção de outro, parecem lógicas as discrepâncias de frames tecnológicos desses grupos, notadamente no que se refere aos pressupostos que mantêm sobre o uso do sistema e de seu gerenciamento. As condições para socialização, interação e negociação moldadas pelo contexto, através da estruturação de relações formais e informais no espaço organizacional, interferem decisivamente nas possibilidades de os grupos atingirem uma situação de consenso negociado (a estabilização e o fechamento de que tratam Pinch e Bijker, 1989) e, em última análise, de congruência. Congruências e incongruências são notadamente influenciadas pelos elementos que originam os frames tecnológicos ou interagem para delineá-los.

\section{As origens dos frames tecnológicos}

Esta pesquisa evidencia que frames tecnológicos originam-se de três dimensões interrelacionadas: características pessoais, características da tecnologia e contexto (ambiente interno e externo), corroborando Orlikowski (1992), que, ao propor o modelo estruturacional da tecnologia, argumenta que a flexibilidade interpretativa de um dado aparato tecnológico não é infinita, mas é limitada pelas características materiais da própria tecnologia, pelo contexto institucional e pelos diferentes níveis de conhecimento e poder dos atores envolvidos com esse artefato tecnológico.

Tome-se o exemplo encontrado na instituição pesquisada em que um mesmo indivíduo desempenha dois papéis na instituição, como analista de sistemas e como aluno. Ao avaliar o sistema contraditoriamente considerando seus papéis na instituição, na condição de profissional da área tecnológica, diz: "é uma excelente ferramenta, um excelente software"; já na condição de aluno, papel secundário desse sujeito na Universidade, afirma: "ele deixa muito a desejar ..., é muito difícil de usar, muito ruim". Ou seja, o sujeito explicita as origens de seus frames tecnológicos. Suas narrativas mostram que conhecimentos, pressupostos e expectativas podem ser frutos de atributos materiais da tecnologia (por exemplo, as interfaces e as funcionalidades a que o sujeito tem acesso em seus diferentes papéis), de traços individuais (por exemplo, formação profissional - enquanto profissional de sistemas, ele considera relevante uma arquitetura tecnológica estável, robusta), e do ambiente (por exemplo, o próprio fato de ocupar diferentes papéis na Universidade e de estabelecer relações com integrantes de ambos os grupos sociais).

As prospecções dos entrevistados sobre as bases de suas opiniões deixam salientes as interações e sobreposições das dimensões que originam os frames. Tais dimensões se manifestam em usos e interpretações da tecnologia de maneiras diferentes, exercendo distintas influências sobre eles, cada uma a seu modo e em variados graus de intensidade.

Um exemplo de como as características pessoais concorrem para modelar um frame tecnológico que repercute nas avaliações individuais do sistema é encontrado na seguinte declaração: 
“eu acho que as coisas, tudo na vida, têm que ter bom senso. E eu sou uma pessoa objetiva e pragmática. Então, se eu sou obrigado a trabalhar com um sistema que não é objetivo e que não anda pelo bom senso, para mim está errado" (professor).

O entrevistado faz relação entre traços de sua personalidade (objetividade e pragmatismo) com características que espera encontrar num sistema de informação. Suas características individuais fundamentam pressupostos (frames) sobre atributos que uma tecnologia deve conter e que, se não atendidos, frustram expectativas (frames).

Sob diferente prisma, um outro depoimento faz referência ao papel das características do agente organizacional na formulação de suas interpretações. Leia-se: "Eu tenho uma visão da área de HC (human-computer), porque eu trabalho com esta área, então eu sou um grande crítico do sistema" (professor). Como o próprio sujeito assume, seu background profissional, seus conhecimentos teóricos e experiências acerca da tecnologia, expandem sua capacidade crítica à medida que lhe permitem observar um conjunto maior de quesitos de julgamento, bem como de melhor qualificá-los. Seu conhecimento técnico, fruto de sua especialização, dá origem a uma série de pressupostos e expectativas de cunho eminentemente prescricional. Ao contrário, quando se analisam declarações de leigos, como é, por exemplo, o caso de alguns alunos, percebe-se um horizonte de análise mais restrito, comumente balizado pela prática de uso do sistema. Ali, o conhecimento formador do frame é empírico e descritivo da relação do usuário com a tecnologia. Se ele, ao interagir com o sistema, enfrenta problemas de acessibilidade a este, tal atributo tende a ser considerado relevante e indicador de baixa qualidade e insucesso daquela tecnologia, ao mesmo tempo em que outros aspectos são desprezados.

A maioria dos usuários cita como principais fontes de seu conhecimento para uso do sistema a própria interação que estabelece com a tecnologia e as relações informais (recebimento de informações por e-mail, busca de informações por conta própria em outros setores, compartilhamento com pares). As características materiais da tecnologia emanam condicionantes para a composição de interpretações pessoais e grupais sobre o aparato tecnológico. A interação indivíduo-tecnologia não apenas é portadora de conhecimentos, como também de pressupostos e expectativas. Coordenadores de curso, por exemplo, pressupõem que não conhecem todas as funções do sistema e que o subutilizam. Um coordenador, quando questionado por que acha que desconhece e subutiliza o sistema acadêmico, recorre à sua interação com a tecnologia, expressando: "Porque de vez em quando a gente descobre uma coisa nova, meio que por acidente, e também porque a gente olha aquela tela, cheia de links e de caminhos, e não tem a menor ideia do que aquilo faz" (coordenador de curso). A ação de "entrar" no sistema e visualizar uma "tela, cheia de links e de caminhos", como diz o coordenador, desencadeia pressupostos de que não conhece o sistema e que o subutiliza, assim como desencadeia a expectativa de conhecê-lo mais.

Além de características pessoais e da tecnologia, a fala dos entrevistados de todos os grupos sociais faz referência ao contexto institucional. Seus frames tecnológicos são também função do ambiente no qual a tecnologia foi desenvolvida, implementada e é operada. O espaço em que se estabelecem as redes de relacionamentos sociais detém características que afetam - favorecem, restringem, determinam - sua composição, seu modus operandi, suas interações. Expectativas e pressupostos em torno da mudança de sistema, no caso estudado, ilustram a influência do contexto (ambiente interno e externo) sobre frames tecnológicos. O depoimento abaixo mostra que, não apenas características materiais da tecnologia, mas também o discurso do fornecedor, ente do ambiente externo, afeta expectativas e pressupostos:

\footnotetext{
"Na época, eu sempre tive boas expectativas porque nas apresentações que eles [os fornecedores] faziam para a reitoria e, normalmente, nas apresentações para a reitoria, eles se esmeravam bastante para vender o produto, porque é ali que se decide, se fica, se não fica. Eu cheguei à conclusão de que realmente era um programa, na minha particularidade, no meu conhecimento rudimentar de sistema, que para nós estava sendo apresentado e era fantástico" (diretor).
}

Similarmente, como indicam outras falas dos entrevistados, como as que seguem, observam-se expectativas e pressupostos frutos do discurso institucional, de conversas informais e de experiências 
anteriores com implementação de sistemas na instituição (contexto-ambiente interno), que remetem aos processos de interação e socialização (citados por Orlikowski \& Gash, 1994) pelos quais se formam e se mantêm frames:

"Eram muitas promessas, umas se concretizaram, outras não. A minha expectativa veio praticamente, ou totalmente, das falas de pessoas que estavam mais diretamente ligadas. Por exemplo: na secretaria diziam que o sistema ia permitir tais coisas, no financeiro diziam que outras coisas poderiam ser realizadas. Então, minhas expectativas foram construídas em cima dessas falas" (coordenador de curso).

"Eu achava isso por causa das experiências anteriores, porque já é o terceiro que a gente utiliza" (coordenador de curso).

Assim como características pessoais, características da tecnologia e propriedades contextuais afetam frames tecnológicos, estes, em sentido oposto, reforçam e/ou modificam atributos materiais da tecnologia, propriedades institucionais e características individuais (Orlikowski, 1992, 2000). Doherty, Coombs e Loan-Clarke (2006) afirmam que as características materiais de um sistema de informação não apenas influenciam a maneira pela qual ele será interpretado pelos stakeholders, mas também que estes, por seu turno, influenciarão como a funcionalidade do sistema é apropriada ou explorada para reforçar interpretações que se tem dele.

Diante das análises e discussões realizadas, um esquema teórico de compreensão de frames tecnológicos é sugerido. A perspectiva interpretativa dos fenômenos sociais, a abordagem qualitativa e o método hermenêutico-dialético amparam epistemologicamente essa proposição, representada pela Figura 1.

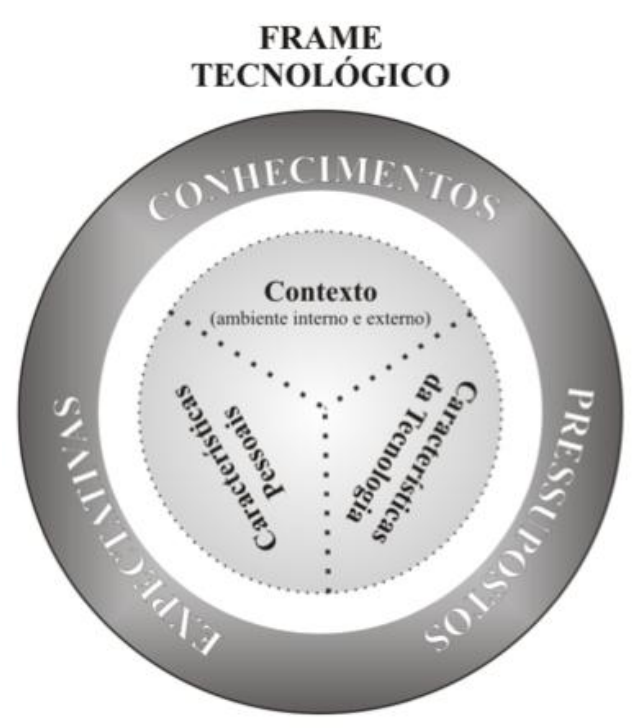

Figura 1. Frame Tecnológico: uma Representação.

Fonte: elaboração dos autores.

Frames tecnológicos, como estruturas coletivas de interpretação da tecnologia, são originários (criados, reforçados, modificados) de características pessoais, contextuais e da própria tecnologia. Nesse núcleo gerador, essas três dimensões sobrepõem-se e influenciam-se mutuamente compondo, como resultantes, os conhecimentos, pressupostos e expectativas, cujos elementos centrais são compartilhados por indivíduos, sobre uma tecnologia. Reciprocamente, frames tecnológicos reforçam e/ou modificam atributos da tecnologia, características contextuais e individuais. 


\section{Considerações Finais}

A estrutura de análise de frames tecnológicos, incluindo os elementos constitutivos de frames (conhecimentos, pressupostos e expectativas), permitiu identificar e analisar a natureza, congruências e incongruências de esquemas interpretativos compartilhados em relação a um sistema de informação. Socialmente construídas, interpretações e avaliações do sistema são fortemente afetadas pelo que os sujeitos conhecem, esperam deste sistema e em que acreditam sobre ele. A partir da interação dinâmica e contínua entre características pessoais, atributos materiais da tecnologia e contexto, conhecimentos, pressupostos e expectativas, assim como a estreita relação que estabelecem entre si, são criados, modificados e reforçados, afetando ações e avaliações do sistema. A interação indivíduotecnologia-contexto manifesta-se em frames tecnológicos de diferentes maneiras e em variados graus de intensidade.

Para fins de investigação do problema de pesquisa proposto, a perspectiva interpretativa e a abordagem qualitativa, em conjunto com os métodos adotados, mostraram-se pertinentes. Acrescentase que a literatura acadêmica sobre frames tecnológicos pouco detalha os procedimentos metodológicos para sua análise. A metodologia empregada nesta pesquisa, em especial os fundamentos e a operacionalização do método hermenêutico-dialético, constituiu-se também em uma das contribuições do trabalho, uma vez que se mostrou relevante para a análise de frames tecnológicos ao permitir a compreensão da subjetividade inerente à interpretação dos sujeitos e dos grupos no contexto social em que se inserem. As descobertas desta pesquisa dificilmente teriam sido alcançadas sob uma perspectiva positivista através de uma abordagem quantitativa, o que reforça a importância do alinhamento dos pressupostos epistemológicos e dos métodos adotados com a proposta e os objetivos da pesquisa.

Outra contribuição tem relação com o contexto em que foi realizada e com o tipo de sistema explorado. Não foram encontrados estudos de análise de frames tecnológicos no contexto universitário, bem como relacionados a sistemas de informação acadêmicos. Esse contexto envolve um amplo conjunto de grupos sociais com papéis bastante distintos (professores, alunos, coordenadores de curso, gestores, colaboradores técnico-administrativos, profissionais de tecnologia, entre outros) além de outras características peculiares, como a duplicidade de papéis exercidos por um mesmo ator, que contribuíram para a compreensão do problema de pesquisa proposto.

Às organizações, esta pesquisa mostrou a influência dos conhecimentos, pressupostos e expectativas sobre as interpretações e avaliações de grupos sociais distintos em relação à tecnologia, assim como os benefícios da análise de frames tecnológicos para compreender julgamentos subjetivos e informais inerentes a contextos sociais. Se há o interesse em compreender a avaliação que sujeitos fazem da tecnologia, sua satisfação ou insatisfação, suas ações, há a necessidade de se compreenderem frames tecnológicos. Conhecimentos, pressupostos e expectativas estão revestidos da interação de características pessoais, tecnológicas e contextuais. Portanto, a compreensão de frames tecnológicos possibilita ainda às organizações identificarem como variáveis e ações institucionais afetam frames. Os frames tecnológicos, suas congruências e incongruências, são socialmente construídos em um contexto, e é neste contexto social que são delineadas as possibilidades de socialização, interação e negociação em torno da tecnologia.

Entende-se que o tema abordado suscita futuros estudos. Visando ao aprofundamento da proposição teórica, procedente da presente pesquisa em torno da estrutura de frames tecnológicos, recomenda-se a realização de pesquisas similares, em outros contextos organizacionais, nãouniversitários, assim como em outras instituições de ensino superior.

Tendo sido observado e ilustrado como as dimensões características pessoais, tecnológicas e contextuais combinam-se para moldar frames tecnológicos, um estudo da relação em sentido oposto como frames tecnológicos afetam estas dimensões - poderá contribuir para o desenvolvimento da teoria de estruturas cognitivas compartilhadas em relação à tecnologia. 
Recomenda-se ainda a adoção da metodologia empregada nesta pesquisa, em especial os fundamentos e a operacionalização do método hermenêutico-dialético, em futuros trabalhos que envolvam a análise de frames tecnológicos.

\section{Artigo recebido em 04.10.2011 Aprovado em 03.04.2012.}

\section{Referências}

Barret, M. (1999). Challenges of EDI adoption for electronic trading in the London Insurance Market. European Journal of Information Systems, 8(1), 1-15. doi: 10.1057/palgrave.ejis.3000313

Berger, P. L., \& Luckmann, T. (2011). A construção social da realidade: tratado de sociologia do conhecimento (33a ed.). Petrópolis: Vozes.

Cole, M., \& Avison, D. (2007). The potential of hermeneutics in information systems research. European Journal of Information Systems, 16(6), 820-833. doi: 10.1057/palgrave.ejis.3000725

Davidson, E. (2002). Technology frames and framing: a socio-cognitive investigation of requirements determination. MIS Quaterly, 26(4), 329-358. doi: 10.2307/4132312

Davidson, E. (2006). A technological frames perspective on information technology and organizational change. Journal of Applied Behavioral Science, 42(1), 23-39. doi: $10.1177 / 0021886305285126$

Davis, F. D., Bagozzi, R. P., \& Warshaw, P. R. (1989). User acceptance of computer technology: a comparison of two theoretical models. Management Science, 35(8), 982-1003. doi: $10.1287 / \mathrm{mnsc} .35 .8 .982$

Doherty, N. F., Coombs, C. R., \& Loan-Clarke, J. (2006). A re-conceptualization of the interpretive flexibility of information technologies: redressing the balance between the social and the technical. European Journal of Information Systems, 15(6), 569-582. doi: 10.1057/palgrave.ejis. 3000653

Howcroft, D., Mitev, N., \& Wilson, M. (2004). What we may learn from the social shaping of technology approach. In L. P. Willcocks \& J. Mingers (Eds.), Social theory and philosophy for information systems (pp. 329-371). Chichester, UK: John Wiley.

Klein, H. K., \& Myers, M. D. (1999). A set of principles for conducting and evaluating interpretive field studies in information systems. MIS Quaterly, 23(1), 67-94. doi: 10.2307/249410

Lin, A., \& Silva, L. (2005). The social and political construction of technological frames. European Journal of Information Systems, 14(1), 49-59. doi: 10.1057/palgrave.ejis.3000521

McGovern, T., \& Hicks, C. (2004). How political processes shaped the IT adopted by a small maketo-order company: a case study in the insulated wire and cable industry. Information \& Management, 42(1), 243-257. doi: 10.1016/j.im.2004.01.004

McLoughlin, I., Badham, R., \& Couchman, P. (2000). Rethinking political process in technological change: socio-technical configurations and frames. Technology Analysis \& Strategic Management, 12(1), 17-37. doi: 10.1080/095373200107210

Minayo, M. C. S. (2008). Hermenêutica-dialética como caminho do pensamento social. In M. Minayo \& S. Deslandes (Orgs.), Caminhos do pensamento: epistemologia e método (2a reimp., pp. 83107). Rio de Janeiro: Fiocruz. 
Moscovici, S. (1961). La psychanalyse: son image et son public. Paris: Puf.

Moscovici, S. (1981). On social representations. In J. P. Forgas (Ed.), Social cognition: perspectives on everyday understanding (pp. 181-209). London: Academic Press.

Nandhakumar J., \& Jones, M. (2002). Development gain? Participant observation in interpretive management information systems research. Qualitative Research, 2(3), 323-341. doi: $10.1177 / 146879410200200303$

Nijland, M. H. (2004). Understanding the use of IT evaluation methods in organizations (Doctoral Dissertation). London School of Economics and Political Science, Department of Information Systems, London, England.

Orlikowski, W. J. (1992). The duality of technology: rethinking the concept of technology in organizations. Organization Science, 3(3), 398-427. doi: 10.2307/2635280

Orlikowski, W. J. (2000). Using technology and constituting structures: a practice lens for studying technology in organizations. Organization Science, 11(4), 404-428. doi: 10.1287/orsc.11.4.404.14600

Orlikowski, W. J., \& Gash, D. C. (1994). Technological frames: making sense of information technology in organizations. ACM Transactions on Information Systems, 12(2), 174-207. doi: $10.1145 / 196734.196745$

Ovaska, P., Rossi, M., \& Smolander, K. (2005). Filtering, negotiating and shifting in the understanding of information system requirements. Scandinavian Journal of Information Systems, 17(1), 31-66.

Pinch, T., \& Bijker, W. E. (1989). The social construction of facts and artifacts: or how the sociology of science and the sociology of technology might benefit each other. In W. E. Bijker, T. P. Hughes, \& T. Pinch (Eds.), The social construction of technological systems: new directions in the sociology and history of technology (pp. 16-50). Cambridge: First MIT Press.

Puri, S. K. (2006). Technological frames of stakeholders shaping the SDI implementation: a case study from India. Information Technology for Development, 12(4), 311-331. doi: 10.1002/itdj.20050

Strauss, A., \& Corbin, J. (2008). Basics of qualitative research: techniques and procedures for developing grounded theory (3rd ed.). Thousand Oaks, CA: Sage Publications.

Symons, V. J. (1991). A review of information systems evaluation: content, context and process. European Journal of Information Systems, 1(3), 205-212. doi: 10.1057/ejis.1991.35

Tan, F. B., \& Gallupe, R. B. (2003). A framework for research into business-IT alignment: a cognitive emphasis. In K. Kangas (Ed.), Business strategies for information technology management (pp. 50-73). Hershey, PA, USA: Idea Group Publishing.

Vaast, E., \& Walsham, G. (2005). Representations and actions: the transformation of work practices with IT use. Information and Organization, 15(1), 65-89. doi: 10.1016/j.infoandorg.2004.10.001

Valsiner, J. (2003). Beyond representations: a theory of enablement. Papers on Social Representations, 12(7), 1-16.

Walsh, J. P. (1995). Managerial and organizational cognition: notes from a trip down memory lane. Organization Science, 6(3), 280-321. doi: 10.1287/orsc.6.3.280

Walsham, G. (2006). Doing interpretive research. European Journal of Information Systems, 15(3), 320-330. doi: 10.1057/palgrave.ejis.3000589 
Weick, K. E. (1973). A psicologia social da organização. São Paulo: Edgard Blucher.

Yin, R. K. (2009). Case study research: design and methods (4th ed.). Thousand Oaks, CA: Sage Publications.

Yoshioka, T., Yates, J. A., \& Orlikowski, W. (2002, January). Community-based interpretive schemes: exploring the use of cyber meetings within a global organization. Proceedings of the Annual Hawaii International Conference on System Sciences (Hicss'02), Washington, DC, USA, 35 . 